\title{
EL ROL DEL EJECUTIVO EN LA PRODUCCIÓN NORMATIVA: EL ECUADOR ENTRE 1979 Y 2016
}

THE ROLE OF THE EXECUTIVE IN NORMATIVE PRODUCTION: ECUADOR BETWEEN 1979 AND 2016

\section{Luis Santiago Llanos Escobar Wladimir García Vinueza**}

Resumen: Este trabajo parte de la siguiente pregunta de investigación: ¿Cómo se ha realizado la aprobación de los proyectos de ley remitidos desde el ejecutivo? Para efectuar este análisis se toma como caso de estudio al Ecuador, entre los años 1979 y 2016, a partir del retorno a la democracia. Para este efecto, a través de una propuesta metodológica cuantitativa de carácter descriptivo, se pretende revisar las formas en las que cada uno de los ejecutivos ecuatorianos ha hecho uso de esta atribución, haciendo énfasis en las condiciones presentes en cada uno de sus gobiernos para este efecto. Por lo tanto, a partir de la recolección y sistematización de información disponible en medios oficiales, se revisa las formas en las que cada ejecutivo ha remitido sus proyectos de ley a la legislatura, evidenciando a primera vista patrones claros que determinen el por qué los ejecutivos consiguen aprobar sus proyectos de ley. El estudio funda sus conclusiones en el funcionamiento de la relación ejecutivo legislativo en el Ecuador, y propone una agenda que permite determinar los elementos causales que coadyuvan para que un ejecutivo un incremento en su nivel de éxito legislativo.

Palabras clave: Ecuador, ejecutivo, legislativo, ley, presidente

Abstract: This work is based on the following research question: how the executive has gotten to approve his bills in the legislative? To carry out this

\footnotetext{
* Investigador de la Pontificia Universidad Católica del Ecuador (Quito, Ecuador). sllanos@campanabogados.com

** Profesor titular de la Pontificia Universidad Católica del Ecuador (Quito, Ecuador). vwgarcia@puce.edu.ec
} 
analysis, Ecuador is taken as a case study between 1979 and 2016, starting from the return to democracy. For this purpose, through a quantitative methodological proposal using descriptive statistics, it is intended to review the ways in which each of the Ecuadorian executives has used his legislative initiative, emphasizing the conditions present in each of their governments for this effect. Therefore, from the collection and systematization of information available in official media, the ways in which each executive has submitted their bills to the legislature are reviewed, evidencing clear patterns which determine why executives get to approve their bills. The study establishes conclusions based on the functioning of the legislative executive relationship in Ecuador; proposes an agenda that allows to determine the causal elements that help an executive to increase his level of legislative success.

Keywords: Ecuador, Executive, Legislative, Law, President

Sumário. I. Introducción. II. La interacción entre el ejecutivo y el legislativo: algunos acercamientos. III. Posibles explicaciones del éxito del ejecutivo. III.1. Mayoría legislativa del partido del ejecutivo. III.2. Capacidades institucionales. III.3. Variables de orden político. III.4. La naturaleza de cada proyecto de ley remitido desde el ejecutivo. IV. La legislatura ecuatoriana. IV.1. Mayoría legislativa y correlación de fuerzas políticas 1979-2015. IV.2. Estrategias usadas por los presidentes ecuatorianos. V. Conclusiones y agenda. Referencias

\section{INTRODUCCIÓN}

El juego político entre funciones del Estado ha sido estudiado ampliamente por parte de la Ciencia Política, en profunda vinculación con el Derecho Constitucional, ya que la parte orgánica de las constituciones determina las competencias y atribuciones generales de los poderes ejecutivo, legislativo y judicial. En particular, uno de los campos de análisis más fértiles de esta tradición es, precisamente, la relación entre las funciones ejecutiva y legislativa. Han sido muchos los autores que, principalmente situados en los Estados Unidos de América, han propuesto modelos teóricos que explican las formas en las que presidente y los congresistas interactúan en el ámbito específico de la aprobación de la ley. Otros tantos han replicado este tipo de teorías al ámbito latinoamericano, extrapolando estudios efectuados en el ámbito del federalismo a los sistemas presidencialistas unitarios.

Este trabajo pretende generar una visión panorámica sobre la aprobación de los proyectos de ley remitidos a la legislatura por parte del ejecutivo en el Ecuador entre los años 1979 y 2016. Para tal efecto, el trabajo 
se realiza en dos etapas. La primera, que analiza los estudios realizados sobre la temática hasta la fecha revisando las propuestas teóricas empleadas por parte de los principales autores sobre la materia. Y la segunda, que expone de forma ordenada y sistemática información relativa a los proyectos de ley presentados y aprobados por cada uno de los presidentes ecuatorianos desde el año 1979, manifestando las características principales tanto de las funciones ejecutiva y legislativa durante los años desde el año 1979.

Con el fin de analizar la temática de manera adecuada, se propone el estudio del caso ecuatoriano por varios motivos. El primero, ya que el análisis de un solo país permite observar y controlar variables contextuales que pudieran no ser identificadas en trabajos que consideren un mayor número de países. El segundo, dado que son pocos los estudios efectuados sobre el Ecuador en lo relativo a los proyectos de ley presentados por parte del presidente, y conviene contar con datos e información completos sobre el país. Y el tercero, dado que la inestabilidad institucional ecuatoriana permite identificar patrones interesantes en la actuación de cada uno de los presidentes ecuatorianos, evidenciando la existencia de casos que confrontan de forma directa las principales conclusiones obtenidas por los estudios sobre la materia.

El mecanismo de análisis propuesto se basa en la generación de estadística de carácter descriptivo, que a través de la sistematización de información disponible en fuentes primarias permite detectar patrones y verificar la forma efectiva en que cada uno de los ejecutivos ecuatorianos ha interactuado con la función legislativa. Las conclusiones permiten observar que los presidentes ecuatorianos, a pesar de contar con contextos aparentemente desfavorables para conseguir la aprobación de sus proyectos de ley, han conseguido aprobar proyectos de ley claves para su gestión, inclusive, en materias económicas y tributarias. Finalmente, se establece una agenda de investigación, que propone estudios futuros haciendo uso de los recursos e información gestados por parte del presente trabajo.

\section{LA INTERACCIÓN ENTRE EL EJECUTIVO Y EL LEGISLATIVO: ALGUNOS ACERCAMIENTOS}

¿Cómo se ha suscitado la aprobación de leyes remitidas a la legislatura por parte del presidente ecuatoriano? Existe una importante tradición de estudios que analizan las relaciones entre el ejecutivo y el legislativo. Uno de los primeros trabajos que pretende revisar las distintas aristas que la relación ejecutivo-legislativo ha tomado es el propuesto por Shugart y Carey (1992). En este trabajo, los autores destacan la importancia del diseño 
institucional al momento de determinar las relaciones entre funciones del estado, y además, en el devenir del régimen democrático. Es decir que, analizan el diseño de la Constitución en el ámbito orgánico, y lo consideran determinante para empujar la balanza a favor de uno u otro poder del Estado. Los autores evidencian la incidencia de las reglas electorales en la determinación de la agenda política, generalmente implementada a través de normas de aplicación general. Además, introducen al debate la existencia de poderes legislativos atribuidos a la función ejecutiva a través del diseño constitucional.

En la misma línea de pensamiento, Mainwaring y Shugart (1997) realizan un estudio situado en América Latina, y dividen las atribuciones conferidas al presidente dentro del proceso legislativo en dos categorías: poderes proactivos y poderes reactivos. Los primeros destacan la iniciativa otorgada al presidente sobre la agenda legislativa. Los segundos, por su parte, se caracterizan por permitir al ejecutivo reaccionar frente a las iniciativas legislativas planteadas por otros autores.

Una de las principales conclusiones del trabajo enunciado en el párrafo que precede es que, en nuestra región, los ejecutivos no requieren de la legislatura para permanecer en el poder. No obstante, sí necesitan de ella para aprobar su agenda de gobierno, que se manifiesta a través de proyectos de ley. Se especifica que, en varios casos, cuando el ejecutivo no logra negociar con la legislatura, podrá utilizar las atribuciones conferidas a nivel constitucional para legislar de forma directa sin necesidad de contar con su colaboración.

Como consecuencia de esto se generan dos lógicas que el ejecutivo puede seguir para conseguir aprobar su agenda de gobierno. La primera, controlar a la función legislativa; y la segunda, pasarla por alto a través de las múltiples atribuciones de carácter institucional que le permiten gobernar por decreto (Negretto, 2003a y 2003b).

La primera estrategia se fundamenta en el poder electoral y las capacidades de negociación efectivas del ejecutivo. Esto se ve reflejado a través de la posibilidad conseguir a través de elecciones una bancada legislativa mayoritaria, siendo primordial la capacidad de determinación de métodos de asignación de escaños. Pero, además, se determina a través de la posibilidad efectiva de negociar con los miembros de la legislatura, generando inclusive una coalición de gobierno que le permita, dentro del ámbito de su curva de indiferencia, contar con colaboradores que persigan una agenda similar a la suya.

La segunda estrategia, por su parte, surge del supuesto de que la primera opción no ha sido viable. En este caso, el presidente, a través de las atribuciones que le han sido conferidas en la Constitución intentará aprobar 
su agenda de gobierno a través de leyes de manera unilateral. En este supuesto, la configuración constitucional es fundamental para entender los mecanismos que podrían ser utilizados por parte del ejecutivo, principalmente, a través de las atribuciones que le permiten ejercer sus poderes legislativos de manera directa.

Complementando las nociones expuestas de forma previa, Cox y Morgenstern (2001) identifican a las asambleas legislativas latinoamericanas como actores primordialmente reactivos, es decir que no hacen ejercicio de su atribución de proponer proyectos de ley, limitándose a responder frente a las propuestas del ejecutivo. Esto se explica, en parte, por el desbalance generado en el diseño institucional de los países latinoamericanos, que favorecen al ejecutivo de forma directa. En este sentido, los autores indican que las legislaturas en América Latina, dependiendo de su conformación y sus intereses, toman una determinada postura frente a los proyectos de ley que son presentados de manera principal por el presidente. Desde esta lógica, generan una tipología relacionada con las posturas tomadas por los congresos frente a distintos tipos de presidentes, pudiendo ser recalcitrantes, negociadores, parroquiales-venales o subordinados (Cox \& Morgenstern, 2001, p. 376). Además, los autores proponen una tipología complementaria, que identifica las diversas relaciones que pueden existir entre presidentes y legislaturas. En concreto, un congreso recalcitrante es aquel que se opone de forma tajante a todas las iniciativas presentadas por parte del ejecutivo. Una legislatura negociadora es aquella que negocia de forma directa con el ejecutivo. Una legislatura parroquial-venal, por su parte, es aquella cuyos miembros pretenden obtener para sí réditos materiales o posiciones de poder. Finalmente, una legislatura subordinada es la que carece de capacidad de respuesta real frente al ejecutivo.

A partir de este razonamiento, los autores parten de una máxima para determinar las formas en que un ejecutivo podrá actuar, determinando que, en todos los casos, el programa de gobierno debe ser solventado de manera exitosa a través de la aprobación de leyes. Por lo tanto, en caso de tener la posibilidad de negociar, o repartir retribuciones entre los congresistas o los partidos de oposición (pork barrel), los titulares de los ejecutivos podrían recurrir a estos medios; pero, en caso de que no exista apertura alguna para efectuar negociaciones, optarán por implementar mecanismos unilaterales, según las atribuciones constitucionales y legales que les son conferidas.

A esta postura se adscriben autores como O'Donnell (1994) y Nino (1996), quienes describen a los legislativos de la región como entes de bajo rendimiento y limitada capacidad. Otros autores, inclusive, han observado que los parlamentos tienen pocas atribuciones para balancear el poder de los ejecutivos en América Latina. (Morgenstern \& Nacif, 2002). La misma idea 
ha sido recogida por Saeigh (2010), quien a través de nueva evidencia empírica identifica a las legislaturas como potenciales apéndices subordinados al poder del ejecutivo.

En este sentido, los ejecutivos deberían contar con una escala de preferencias modelada de la siguiente manera:

Opción 1: conseguir una mayoría legislativa afín, ya sea a través del sistema electoral. Este hecho parte del supuesto de que el partido al que el presidente pertenece cuenta con un importante nivel de disciplina partidista.

Opción 2: obtener una mayoría legislativa de coalición a través de la sesión efectiva de espacios de poder, conformando de esta forma una coalición de gobierno.

Opción 3: hacerse de una mayoría temporal para aprobar determinados proyectos de ley, sin necesidad de conformar una coalición de gobierno.

Opción 4: conseguir la aprobación de sus proyectos de ley presionando a la legislatura a través de las atribuciones institucionales.

Determinar el orden de prelación en el que las opciones anteriores se aplican resulta en todo caso complejo, debido a que podrían depender de varios elementos. Sin embargo si el presidente, por ejemplo, cuenta con grandes atribuciones institucionales, sus incentivos para la negociación serán bajos, dado que, en todos los casos, la capacidad de implementación unilateral real fungiría como un incentivo negativo para incurrir en costos inherentes a los procesos de negociación.

En todo caso, la formulación de esta escala de preferencias será útil al momento de contar con información sistematizada, ya que podría constituirse como una categoría analítica con limitado poder predictivo para entender lo que un ejecutivo podrá hacer. De esta forma, se aporta para el entendimiento de las relaciones ejecutivo legislativo.

Este tipo de previsiones generan aparentemente un inconveniente a partir del marco constitucional inherente a los sistemas presidencialistas que caracterizan a América Latina. Esto, principalmente, por el hecho de que los diseños constitucionales podrían encontrarse desbalanceados a favor de los ejecutivos, generando, además, la imposibilidad de funcionamiento óptimo al sistema de frenos y contrapesos, fundamental para la existencia de la división de poderes.

En este contexto, existen múltiples estudios que han procurado entender las formas en las que los ejecutivos consiguen aprobar sus proyectos de ley, verificando a través del estudio de casos empíricos la forma en que los ejecutivos han interactuado con la legislatura a través de la aprobación de sus proyectos de ley. 
Bajo esta perspectiva, las principales explicaciones propuestas para el éxito del ejecutivo son, principalmente las atribuciones constitucionales otorgadas al presidente, y las variables de naturaleza política que pueden determinar elementos favorables o no para la aprobación de la agenda legislativa propuesta desde el ejecutivo.

\section{POSIBLES EXPLICACIONES DEL ÉXITO DEL EJECUTIVO}

Para tratar este apartado de manera ordenada se ha ajustado la discusión de la literatura especializada en la materia, a partir de las posibles explicaciones propuestas por los autores para el estudio de la temática. Así, se ha articulado la discusión en cuatro grupos de variables propuestas desde la literatura, que son las siguientes: mayoría legislativa del partido del ejecutivo, capacidades institucionales de la función ejecutiva, variables políticas y naturaleza de los proyectos de ley propuestos por el ejecutivo.

\section{III.1. Mayoría legislativa del partido del ejecutivo}

Algunos estudios clásicos, como el de Linz (1990), observan que el fundamento de la aprobación de los proyectos de ley remitidos desde el ejecutivo es la obtención de una mayoría legislativa afín. Esto resulta lógico por cuanto se prevé que los miembros de la bancada oficialista dentro de la asamblea tienen un programa similar al del presidente. No obstante, esta percepción parte del supuesto de que existe disciplina partidista, cuestión que no debe darse por sentada al momento de realizar trabajos que coordinen lo teórico con lo empírico. Es decir, el efecto de este tipo de variables podría verse condicionado por la existencia de otros requisitos.

A pesar de que existen críticas a esta postura, son varios los estudios que confirman esta hipótesis (Mainwaring, 1993; Deheza, 1994; Mainwaring \& Shugart, 1997; Steger, 1997; Amorim \& Cox, 1998; García Montero, 2007; García Montero, 2009; Santos, Pérez Liñan \& García Montero, 2014), indicando que, en efecto, un presidente con una bancada de menor tamaño en la legislatura experimentará mayores dificultades para conseguir aprobar sus proyectos de ley, controlar la agenda legislativa, y además, poder implementar su programa de gobierno.

Considerando que el número de legisladores afines al primer mandatario importa, como se demuestra en los trabajos enunciados de forma previa, es claro que los presidentes intentarán contar con un número de legisladores afines amplio. Este hecho les motiva a conformar coaliciones dentro de la legislatura, siguiendo lógicas de negociación, como indican varios autores (Altman, 2001; Chasquetti, 2001; 2008; Amorim \& Cox, 
1998; Pérez Liñán, 2000). No obstante, en algunos casos, no contar con una mayoría en la legislatura afín genera que los presidentes incurran en procedimientos de negociación concretos e individuales para la aprobación de proyectos de ley específicos, mediante la formación de lo que Mejía (2004) denominada "coaliciones fantasma". Este tipo de coaliciones, de hecho, persigue la aprobación de proyectos de ley concretos. En ningún caso busca generar una bancada legislativa permanente, o una coalición de gobierno que permita un nivel de cooperación estable.

Existen otros estudios centrados principalmente en la legislatura estadounidense. Han demostrado que si bien contar con una mayoría de legisladores por parte del presidente contribuye para la aprobación de sus proyectos de ley en la legislatura, se suscitan momentos en los que a pesar de contar con un congreso contrario, sus iniciativas legales se aprueban sin cambios ni trabas dentro del legislativo (Mayhew, 2005). Estas lógicas evidencian la existencia de procesos de negociación subyacentes, o en su defecto, de otro tipo de explicaciones que favorecen que la función ejecutiva logre aprobar sus proyectos de Ley dentro de la legislatura.

En todo caso, la literatura especializada ha propuesto varias explicaciones para que esto ocurra. Por ejemplo, la posibilidad de cooptación de legisladores mediante la asignación de recursos y beneficios, en concordancia con lo previsto por Cox y Morgenstern (2001), con referencia a las legislaturas de carácter parroquial venal. Pero, por otra parte, también puede pensarse que los presidentes, a través de los poderes institucionales que ostentan, principalmente aquellos encaminados al establecimiento de la agenda a tratarse en la legislatura (Alemán \& Navia, 2009), pueden conseguir la aprobación de sus proyectos de ley. En este sentido, cobra relevancia el análisis de los distintos actores que cuentan con iniciativa legislativa, pues su interacción puede entenderse como un juego en donde uno de los actores podría llevarse todos los beneficios, o bien, un juego en el que la cooperación es el mejor camino para los jugadores.

\section{III.2. Capacidades institucionales}

Varios estudios se han enfocado en analizar las capacidades institucionales atribuidas al presidente como factores determinantes para la aprobación de sus iniciativas legales dentro de la legislatura (Mainwaring \& Shugart, 1997; Mejía, 2002, 2004; Tsebelis \& Alemán, 2005; García Montero, 2007, 2009; Alemán \& Navia, 2009, Santos, Pérez Liñán \& García Montero, 2014). En este sentido, son varias las atribuciones otorgadas a favor del ejecutivo que deben ser analizadas, por ejemplo: la capacidad de veto, la capacidad de declaratoria de urgencia en un proyecto de ley, la 
capacidad de decreto de estado de excepción, o la iniciativa legislativa exclusiva sobre materias determinadas. Desde esta lógica, se entiende que, a medida que las capacidades atribuidas al presidente a nivel normativo son superiores, éste tendrá mayores posibilidades de actuar de manera unilateral frente a la legislatura. Además, a mayor capacidad institucional, el ejecutivo podría tener mayor fortaleza para presionar al poder legislativo. Para algunos autores, esta capacidad es determinante, debido a que permiten la generación de lo que, para Cox y Morgenstern se denomina un presidente imperial, que actúa de forma unilateral, haciendo un by pass de la legislatura, e implementando su agenda de gobierno de forma directa. Evidentemente, este caso no pretende ser observado dentro del presente estudio, dado que se pretende observar la forma de interacción del ejecutivo y el legislativo específicamente en el ámbito de la aprobación de las iniciativas legales.

En este sentido, dentro de este tipo de estudios se ha analizado las capacidades institucionales otorgadas a favor del presidente de la República como claves para la aprobación de sus proyectos de Ley. Una estrategia utilizada por Mejía $(2002,2004)$, por ejemplo, es la determinación de una gran cantidad de leyes identificadas como económicas urgentes durante el primer año de gobierno del presidente León Febres Cordero en el Ecuador, lo que le permitió aprobar un $65 \%$ de sus iniciativas legislativas durante su primer año de gobierno (Burbano \& Rowland, 1998). De esta forma, se determina que las capacidades institucionales son un elemento clave para la determinación de la capacidad efectiva del presidente de la República para obtener la aprobación de sus proyectos de ley. No obstante, es importante tomar en cuenta que este elemento no puede ser considerado como único y determinante, debido a que, según Burbano y Rowland (1998) y Mejía (2002), durante los siguientes años de su gobierno - posteriores al primero-, el presidente Febres Cordero tuvo mayor resistencia dentro de la legislatura para la aprobación de sus proyectos de ley.

Varias formas de analizar la incidencia de las capacidades institucionales han sido propuestas desde la literatura especializada. Alemán y Navia (2009) y García Montero (2009), identifican que un presidente con mayores capacidades institucionales podrá presionar de manera efectiva al legislativo para la aprobación de sus proyectos de ley. Sin embargo, debemos tomar en cuenta que la capacidad institucional no puede tomarse como un elemento único y aislado para explicar el mayor éxito legislativo de un presidente. En este sentido, un ejecutivo con capacidades institucionales grandes bien podría gobernar directamente, por decreto, o decreto ley, sin necesidad de entrar en lógicas de negociación o disputa con la Asamblea Legislativa. No obstante, se debe tener en cuenta que existe reserva de ley para el tratamiento de determinados temas. En este caso, el 
presidente se encuentra en la necesidad de iniciar procedimientos de negociación con la legislatura y sus actores, para procurar la aprobación e implementación de su agenda de gobierno.

En esta misma línea, uno de los principales antecedentes para determinar la incidencia de las capacidades institucionales en la aprobación de los proyectos de ley de los ejecutivos es el propuesto por García Montero (2009). Dentro de este trabajo, la autora genera el denominado índice IPIL, que consiste en un indicador relativo al poder del ejecutivo en base a 14 criterios, organizados en 5 categorías. Este indicador se concibe con relación a las capacidades institucionales del ejecutivo aplicables en el proceso legislativo de formación de la ley. De esta forma, el índice IPIL, permite una evaluación general de las atribuciones del ejecutivo con relación al proceso legislativo. No obstante, existen otro tipo de mediciones relativas al poder del ejecutivo, vinculadas principalmente a los estudios sobre hiperpresidencialismo, donde se mide la potencia del ejecutivo de forma general y sin un énfasis determinado en el proceso legislativo (Valdivieso \& Rivera, 2015). En todo caso, este último tipo de indicadores no han sido utilizados en estudios relativos a la aprobación de los proyectos de ley del ejecutivo.

Por otra parte, el estudio propuesto por García Montero (2009) en el ámbito de América Latina a través de la implementación del denominado índice IPIL como variable explicativa evidencia resultados favorables. De esta forma, identifica que, a medida que un ejecutivo cuenta con mayores capacidades institucionales, es decir, con un índice IPIL más alto, la probabilidad de aprobar sus proyectos de ley incrementa.

Por su parte, el estudio de Alemán y Navia (2009) indica que, efectivamente, las capacidades institucionales del ejecutivo son importantes al momento de aprobar sus proyectos de ley. En concreto, su estudio analiza la capacidad del ejecutivo de catalogar a los proyectos de ley presentados por el presidente como económicos urgentes. A través de esta designación, el presidente consigue que sus proyectos de ley se sujeten a un procedimiento especial, que favorece la aprobación de sus proyectos de ley en la legislatura.

En todo caso, la literatura sobre la temática determina que este tipo de variables son importantes al momento de observar un incremento en las capacidades efectivas del primer mandatario para obtener la aprobación de sus proyectos de ley. Sin embargo, varios de los autores que han utilizado variables explicativas de esta naturaleza (Mejía, 2002; Tsebelis \& Alemán, 2005; Alemán \& Navia, 2009; García Montero, 2009; Santos, Pérez Liñán \& García Montero, 2014), proponen un grupo de variables complementarias que también inciden de forma representativa en la aprobación de las iniciativas legales remitidas desde la función ejecutiva, como por ejemplo, las variables de orden político. Son analizadas a continuación. 
Sin embargo, debe tomarse en cuenta que existen otro tipo de variables institucionales no analizadas en este trabajo que podrían ser relevantes. Un ejemplo claro es la conformación de la legislatura, cuya estructura y funcionamiento puede influir en aumento o disminución de la capacidad del ejecutivo de aprobar proyectos de ley dentro de la legislatura.

\section{III.3. Variables de orden político}

La literatura ha identificado una serie de variables de índole político como potencialmente explicativas de la aprobación de los proyectos de ley remitidos a la legislatura por parte del presidente (García Montero, 2009; Alemán \& Navia, 2009; Santos, Pérez Liñán \& García Montero, 2014). Entre las principales explicaciones propuesta se puede destacar el nivel de fragmentación partidista, la distancia ideológica entre el partido mayoritario del congreso y el ejecutivo, la distancia ideológica entre partidos de la Asamblea Legislativa y el calendario electoral (Periodo luna de miel ${ }^{1}$ ). Otro tipo de estudios relativos al Congreso de los Estados Unidos han propuesto variables explicativas de distinta naturaleza, como por ejemplo la capacidad de respuesta de las legislaturas, es decir, sus capacidades institucionales y políticas, la división de funciones del estado en su plano formal y material, el calendario electoral y la popularidad presidencial (Edwards, 1989; Bond \& Fleisher,1990; Covington, Wrighton \& Kinney,1995). No obstante, es importante tomar en cuenta que las características del sistema político norteamericano generan que los estudios enfocados en este contexto no sean necesariamente extrapolables a otros, debido a que la configuración institucional de este país es distinta, y además, las características de su sistema político son diferentes a las de América Latina. Uno de los principales elementos que pueden ayudarnos a identificar estas circunstancias son: su sistema bipartidista, su método de asignación de escaños y su diseño institucional.

Uno de los principales estudios relativos a variables de orden político es el efectuado por Alemán y Navia (2009) a través del cual se analiza a la legislatura chilena en su interacción frente a la función ejecutiva. En este caso, los autores proponen tres variables explicativas claves: las capacidades institucionales del ejecutivo, manifestadas como la capacidad de presentar

\footnotetext{
${ }^{1}$ El periodo "luna de miel" ha sido definido por Alemán y Navia (2009) como el término de un año contado a partir de que un presidente asume sus funciones. Se le imputa un determinado nivel de causalidad como explicación de la capacidad efectiva del presidente para aprobar sus proyectos de ley en la legislatura, debido a que cuando el presidente asume sus funciones cuenta con su máximo nivel de popularidad durante toda su gestión, y además, tiene muchos incentivos para cumplir sus ofertas de campaña.
} 
proyectos de ley catalogados como económicos urgentes — analizada en párrafos anteriores - ; la vigencia del periodo luna de miel y adicionalmente una variable contextual, determinada como la popularidad del presidente al momento de aprobar sus proyectos de ley en el congreso. Las hipótesis de los autores se confirman de forma parcial, determinado que tanto el periodo luna de miel, así como las prerrogativas del ejecutivo (capacidades institucionales) son efectivamente relevantes para explicar su éxito. Por otra parte, los resultados obtenidos por los autores no permiten verificar su hipótesis relacionada a la popularidad del ejecutivo, de manera contradictoria con los estudios efectuados en los Estados Unidos que se enuncian previamente. En todo caso, esta última hipótesis es de difícil medición, dado que el levantamiento de datos homogéneos de popularidad que sean comparables se complica en la realización de estudios comparados o en periodos de tiempo largos.

No obstante, el análisis de las variables políticas ha obligado a los autores a observar periodos de tiempo largos, con el fin de otorgar valores fiables a sus mediciones. Esta situación ha generado que, de hecho, la materia, tipo y contenido específico de cada uno de los proyectos de ley remitidos desde el ejecutivo no puedan ser analizados de forma profunda. Desde esta perspectiva, es conveniente analizar otro tipo de estudios relativos a esta temática generados por diversos autores, dentro de los cuales se analizan otro tipo de variables explicativas.

Por su parte, existen otras variables de orden político relevantes identificadas por García Montero (2009), como la distancia ideológica entre partidos de la legislatura, el nivel de fragmentación partidista, la distancia ideológica entre el presidente y el partido mayoritario de la legislatura entre otras. Efectivamente, la autora demuestra que este tipo de variables son relevantes al momento de determinar un incremento de las posibilidades de aprobación de los proyectos de ley remitidos desde el ejecutivo a la legislatura. La incidencia de este tipo de variables de hecho, es confirmada de manera posterior dentro del estudio realizado por parte de Santos, Pérez Liñán y García Montero (2014).

\section{III.4. La naturaleza de cada proyecto de ley remitido desde el ejecutivo}

Dentro de los múltiples estudios sobre América Latina, existen importantes estudios efectuados para Brasil (Amorim, Cox \& McCubbins, 2003; Limongi \& Figuereido, 2000). En el caso del primer estudio los autores evidencian las fluctuaciones suscitadas en las mayorías parlamentarias, observando sus implicaciones con relación al control de la agenda legislativa. En cuanto al segundo estudio, se efectúa un análisis 
relativo a la disciplina partidista y su influencia en determinar el incremento en el éxito legislativo de los presidentes brasileños. A partir de esto, los autores verifican sus estudios utilizando un campo específico de proyectos de ley para detectar la relevancia de su materia para su posterior aprobación.

Otro de los claros ejemplos de este tipo de estudios es el efectuado por parte de Alemán y Navia (2009), quienes efectúan un análisis de las iniciativas legislativas presentadas por parte del ejecutivo respecto de campos específicos de la política pública considerada altamente relevante. No obstante, su forma de proceder no implica la determinación de la materia del proyecto de ley como una variable independiente que forme parte del modelo utilizado. En todo caso, se verifica que esta temática ha sido estudiada de manera incidental, sin que exista una importante tradición de estudios que haya estudiado esta cuestión y el estudio es destacable por la incorporación de esta temática al análisis.

En síntesis, los autores mencionados en el párrafo anterior analizan la aprobación de los proyectos de ley con base en campos específicos de la política pública. Desde esta perspectiva, se concluye que la posibilidad efectiva de aprobación de los proyectos de ley remitidos desde el ejecutivo se relativiza con relación a la materia del proyecto de ley particular. En especial, sus resultados indican que proyectos de ley en materia económica y reformas constitucionales son más complejas de aprobar por parte de los ejecutivos.

Por lo tanto, uno de los principales aportes de este trabajo será incluir la materia del proyecto de ley como una variable explicativa para su aprobación o no. A partir de esta lógica, se pretende verificar que existirán campos de la política pública de relevancia baja, en cuyo caso el presidente podría obtener una aprobación poco problemática de sus iniciativas legislativas. Pero, por otra parte, también se concibe la existencia de materias que, por su naturaleza (como proyectos de ley en el campo económico) podrían ser campos que ameritarían mayores esfuerzos de parte del ejecutivo para conseguir su aprobación.

\section{LA LEGISLATURA ECUATORIANA}

Como su nombre indica, la función legislativa se encarga de legislar. Bajo esta lógica, le corresponde aprobar los proyectos de ley presentados por los distintos actores a los que el marco institucional les otorga iniciativa legal. El procedimiento seguido para este efecto en el Ecuador, desde el año 1979, se ha mantenido relativamente establece, y puede resumirse en los siguientes pasos: 1) presentación de la ley; 2) calificación de la misma; 3) discusión en primer debate; 4) discusión en segundo de debate; 5) 
aprobación o archivo; 6) veto o sanción presidencial; y, 7) publicación. La certeza sobre el proceso legislativa es importante, pues permite determinar el momento en que un presidente consigue tener éxito, y cuáles son los momentos donde podrían utilizar sus recursos políticos e institucionales para procurar obtener la aprobación de sus proyectos de ley.

\section{IV.1. Mayoría legislativa y correlación de fuerzas políticas 1979-2015}

Son varios los elementos que pueden tener influencia sobre la aprobación de un determinado proyecto de ley. Uno de los principales es la correlación de fuerzas políticas a nivel de la función legislativa.

El caso ecuatoriano resulta interesante para su análisis debido a varios elementos propios de su contexto, entre los cuales se pueden destacar: la inestabilidad institucional experimentada desde el retorno a la democracia, la existencia de doce presidentes de características muy diversas durante un periodo de apenas 28 años, y la existencia de condiciones diversas dentro de las legislaturas a lo largo a partir del retorno a la democracia. Pero además, la corta vida de los partidos políticos, y los cambios generales en el panorama dan cuenta de importantes niveles de inestabilidad, que permiten analizar legislaturas con una composición totalmente distinta dentro de un periodo de tiempo relativamente corto. La evidencia de esto se encuentra en la inexistencia de un partido que haya contado con presencia permanente y homogénea dentro de la legislatura durante el periodo de tiempo analizado.

En este sentido, puede verificarse que la legislatura ecuatoriana no ha tenido un funcionamiento homogéneo. Pero a pesar de ello, se presentan cuestiones interesantes. Por ejemplo, Mejía (2002, 2004) verifica que, a pesar de que ninguno de los presidentes ecuatorianos, a excepción de Rafael Correa, ha contado con una mayoría propia en la legislatura, varios presidentes lograron aprobar proyectos de ley emblemáticos, que les han permito introducir importantes reformas políticas, económicas y jurídicas.

A partir del año 1979, ningún presidente ecuatoriano ha contado con una mayoría legislativa propia, salvo Rafael Correa Delgado durante sus dos últimos periodos de gobierno. Otro caso interesante es el de Rodrigo Borja Cevallos, quien, durante su primer año de gobierno contó con una mayoría de coalición, en una alianza de su partido, la Izquierda Democrática, con Unión Demócrata Cristiana (DP-UDC) y el Frente Amplio de Izquierda (FADI). Posteriormente, la mayoría de coalición disminuye, pues que, para el segundo año de gobierno del presidente Borja, la DP-UDC decide salir de la coalición dejando al partido de Borja sin mayoría legislativa, pero sí con una bancada representativa dentro de la legislatura, equivalente al 45,07\% del total de asientos en el Congreso Nacional. 
Salvo los casos excepcionales que se indican, los presidentes ecuatorianos no han tenido una posición cómoda dentro de la legislatura. Y así, se puede verificar que la mayoría oficialista, a lo largo de los años ha sido cambiante. Esto se expone de forma precisa en el siguiente cuadro:

Tabla 1: Tamaño de las bancadas legislativas 1979 - 2016

\begin{tabular}{|c|c|c|c|c|}
\hline \multirow{2}{*}{$\begin{array}{l}\text { Periodo temporal de } \\
\text { cambio de coalición }\end{array}$} & \multirow{2}{*}{ Presidente } & \multirow{2}{*}{$\begin{array}{l}\text { Partido del } \\
\text { presidente }\end{array}$} & \multicolumn{2}{|c|}{ Bancada legislativa } \\
\hline & & & propia & de coalición ${ }^{2}$ \\
\hline Ago/1979-Ago/1980 & Jaime Roldós A. & CFP & $36,23 \%$ & $66,67 \%$ \\
\hline Ago/1980 & Jaime Roldós A. & CFP & $36,23 \%$ & $43,48 \%$ \\
\hline Sep/1980-Abr/1981 & Jaime Roldós A. & CFP & $36,23 \%$ & $\mathrm{n} / \mathrm{a}$ \\
\hline May/1981-May/1982 & $\begin{array}{l}\text { Oswaldo Hurtado } \\
\text { Larrea }\end{array}$ & $\begin{array}{l}\text { Democracia Popular / } \\
\text { UDC }\end{array}$ & $7,25 \%$ & $26,09 \%$ \\
\hline Jun/1982-Ene/1983 & $\begin{array}{l}\text { Oswaldo Hurtado } \\
\text { Larrea }\end{array}$ & $\begin{array}{l}\text { Democracia Popular / } \\
\text { UDC }\end{array}$ & $7,25 \%$ & $57,97 \%$ \\
\hline Feb/1983-Ago/1984 & $\begin{array}{l}\text { Oswaldo Hurtado } \\
\text { Larrea }\end{array}$ & $\begin{array}{l}\text { Democracia Popular / } \\
\text { UDC }\end{array}$ & $7,25 \%$ & $\mathrm{n} / \mathrm{a}$ \\
\hline Ago/1984-Ago/1986 & León Febres Cord. & Social Cristiano & $12,68 \%$ & $29,58 \%$ \\
\hline Sep/1986-Jul/1988 & León Febres Cord. & Social Cristiano & $16,90 \%$ & $\mathrm{n} / \mathrm{a}$ \\
\hline Ago/1988-Jul/1989 & Rodrigo Borja C. & Izquierda Democrática & $42,25 \%$ & $54,93 \%$ \\
\hline Ago/1989-Jun/1990 & Rodrigo Borja C. & Izquierda Democrática & $42,25 \%$ & $45,07 \%$ \\
\hline Jul/1990 & Rodrigo Borja C. & Izquierda Democrática & $42,25 \%$ & $\mathrm{n} / \mathrm{a}$ \\
\hline Ago/1990-Jul/1992 & Rodrigo Borja C. & Izquierda Democrática & $19,44 \%$ & $\mathrm{n} / \mathrm{a}$ \\
\hline Ago/1992-Ago/1994 & $\begin{array}{l}\text { Sixto Durán } \\
\text { Ballén }\end{array}$ & $\begin{array}{l}\text { Unidad Republicana / } \\
\text { Partido Conservador } \\
\text { Ecuatoriano }\end{array}$ & $23,37 \%$ & $50,65 \%$ \\
\hline Ago/1994-Jul/1995 & $\begin{array}{l}\text { Sixto Durán } \\
\text { Ballén }\end{array}$ & $\begin{array}{l}\text { Unidad Republicana / } \\
\text { Partido Conservador } \\
\text { Ecuatoriano }\end{array}$ & $14,28 \%$ & $56,92 \%$ \\
\hline Ago/1995-Sep/1995 & $\begin{array}{l}\text { Sixto Durán } \\
\text { Ballén }\end{array}$ & $\begin{array}{l}\text { Unidad Republicana / } \\
\text { Partido Conservador } \\
\text { Ecuatoriano }\end{array}$ & $14,28 \%$ & $\mathrm{n} / \mathrm{a}$ \\
\hline Oct/1995-Jul/1996 & $\begin{array}{l}\text { Sixto Durán } \\
\text { Ballén }\end{array}$ & $\begin{array}{l}\text { Unidad Republicana / } \\
\text { Partido Conservador } \\
\text { Ecuatoriano }\end{array}$ & $14,28 \%$ & $44,62 \%$ \\
\hline Ago/1996-Nov/1996 & $\begin{array}{l}\text { Abdalá Bucaram } \\
\text { Ortiz }\end{array}$ & $\begin{array}{l}\text { Partido Roldosista } \\
\text { Ecuatoriano }\end{array}$ & $24,39 \%$ & $64,29 \%$ \\
\hline Dic/1996-Ene/1997 & $\begin{array}{l}\text { Abdalá Bucaram } \\
\text { Ortiz }\end{array}$ & $\begin{array}{l}\text { Partido Roldosista } \\
\text { Ecuatoriano }\end{array}$ & $24,39 \%$ & $30 \%$ \\
\hline Feb/1997-Jul/1998 & Fabián Alarcón R. & Frente Radical Alfarista & $2,44 \%$ & $\mathrm{n} / \mathrm{a}$ \\
\hline
\end{tabular}

${ }^{2}$ Cuando no se reporta la existencia de una coalición legislativa, el valor correspondiente a bancada legislativa de coalición se toma del valor de la bancada legislativa propia. 
Luis Santiago Llanos Escobar \& Wladimir García Vinueza

\begin{tabular}{|c|c|c|c|c|}
\hline Ago/1998-Feb/1999 & $\begin{array}{l}\text { Jamil Mahuad } \\
\text { Witt }\end{array}$ & $\begin{array}{l}\text { Democracia Popular } \\
\text { UDC }\end{array}$ & $30,58 \%$ & $56,20 \%$ \\
\hline Mar/1999-Jun/1999 & $\begin{array}{l}\text { Jamil Mahuad } \\
\text { Witt }\end{array}$ & $\begin{array}{l}\text { Democracia Popular } \\
\text { UDC }\end{array}$ & $30,58 \%$ & $47,93 \%$ \\
\hline Jul/1999-Sep/1999 & $\begin{array}{l}\text { Jamil Mahuad } \\
\text { Witt }\end{array}$ & $\begin{array}{l}\text { Democracia Popular } \\
\text { UDC }\end{array}$ & $30,58 \%$ & $\mathrm{n} / \mathrm{a}$ \\
\hline Oct/1999-Nov/1999 & $\begin{array}{l}\text { Jamil Mahuad } \\
\text { Witt }\end{array}$ & $\begin{array}{l}\text { Democracia Popular } \\
\text { UDC }\end{array}$ & $30,58 \%$ & $50,41 \%$ \\
\hline Dic/1999-Ene/2000 & $\begin{array}{l}\text { Jamil Mahuad } \\
\text { Witt }\end{array}$ & $\begin{array}{l}\text { Democracia Popular } \\
\text { UDC }\end{array}$ & $30,58 \%$ & $\mathrm{n} / \mathrm{a}$ \\
\hline Feb/2000-May/2000 & Gustavo Noboa B. & Ninguno & $0 \%$ & $55,37 \%$ \\
\hline Jun/2000-Jul/2000 & Gustavo Noboa B. & Ninguno & $0 \%$ & $69,42 \%$ \\
\hline Ago/2000-Ene/2001 & Gustavo Noboa B. & Ninguno & $0 \%$ & $57,02 \%$ \\
\hline $\mathrm{Feb} / 2001-\mathrm{Abr} / 2001$ & Gustavo Noboa B. & Ninguno & $0 \%$ & $\mathrm{n} / \mathrm{a}$ \\
\hline Abr/2001-May/2001 & Gustavo Noboa B. & Ninguno & $0 \%$ & $70,25 \%$ \\
\hline Jun/2001-Dic/2002 & Gustavo Noboa B. & Ninguno & $0 \%$ & $\mathrm{n} / \mathrm{a}$ \\
\hline Ene/2003-Jul/2003 & Lucio Gutiérrez B. & Sociedad Patriótica & $9 \%$ & $20 \%$ \\
\hline Ago/2003-Abr-2004 & Lucio Gutiérrez B. & Sociedad Patriótica & $9 \%$ & $50 \%$ \\
\hline May/2004-Sep/2004 & Lucio Gutiérrez B. & Sociedad Patriótica & $9 \%$ & $34 \%$ \\
\hline Oct/2004 & Lucio Gutiérrez B. & Sociedad Patriótica & $9 \%$ & $\mathrm{n} / \mathrm{a}$ \\
\hline Nov/2004-Abr/2005 & Lucio Gutiérrez B. & Sociedad Patriótica & $9 \%$ & $15 \%$ \\
\hline May/2005-Dic/2006 & Alfredo Palacio G. & Ninguno & $0 \%$ & $27 \%$ \\
\hline Ene/2007-Nov/2007 & Rafael Correa D. & Mov. Alianza País & $0 \%$ & $\mathrm{n} / \mathrm{a}$ \\
\hline $\mathrm{Dic} / 2007-\mathrm{Oct} / 2008^{3}$ & Rafael Correa D. & Mov. Alianza País & $61,57 \%$ & $67,69 \%$ \\
\hline Nov/2008-Jul/20094 & Rafael Correa D. & Mov. Alianza País & $60,53 \%$ & $67,11 \%$ \\
\hline Ago/2009-Abr/2013 & Rafael Correa D. & Mov. Alianza País & $47,58 \%$ & $54,84 \%$ \\
\hline May/2013-Mar/2015 & Rafael Correa D. & Mov. Alianza País & $72,99 \%$ & $76,64 \%$ \\
\hline Mar/2015 & Rafael Correa D. & Mov. Alianza País & $72,99 \%$ & $\mathrm{n} / \mathrm{a}$ \\
\hline
\end{tabular}

Fuente: Elaboración propia con base en Burbano \& Rowland (1998) y Valdivieso (2015)

Esta evidente carencia de mayorías propias dentro de la legislatura ecuatoriana ha obligado a los presidentes a buscar la formación de coaliciones con el fin de aprobar sus agendas de gobierno dentro de la legislatura. En general, este fenómeno puede verificarse en la formación y desintegración de coaliciones de forma reiterada. Por otra parte, la carencia

\footnotetext{
${ }^{3}$ Asamblea Constituyente convocada por Rafael Correa Delgado, al disolver a la Asamblea Legislativa utilizando las prerrogativas institucionales que le confirió la Constitución ecuatoriana de 1998.

${ }^{4}$ Se hace referencia al denominado Congresillo, que ejerció la Función Legislativa hasta la convocatoria a elecciones del año 2009.
} 
de una mayoría legislativa propia ha generado la eventual existencia de pagos ilegítimos para la conformación de las denominadas coaliciones fantasma de lo que Mejía (2002, 2004).

Por otra parte, debe tomarse en cuenta que en los casos en los que el presidente no cuente con una mayoría legislativa afín, podrá recurrir a mecanismos de presión unilateral en base a sus atribuciones institucionales. De esta forma, por ejemplo, puede observarse que León Febres Cordero, durante el primer año de su mandato recurrió a remitir a la Asamblea Nacional ingentes cantidades de proyectos de ley catalogados como económicos urgentes, de manera que, la Asamblea Nacional, ante la imposibilidad de conocer todos los proyectos de ley dentro del términos de treinta días determinado en la Constitución ecuatoriana de 1979, omitía un pronunciamiento, y en consecuencia, los proyectos de ley eran aprobados como decretos ley (Mejía, 2002, 2004).

\section{IV.2. Estrategias usadas por los presidentes ecuatorianos}

Dentro de la legislatura ecuatoriana existen varios vasos dignos de análisis. Uno de ellos es el de León Febres Cordero, que ejemplifica un claro caso de utilización de mecanismos de unilaterales para aprobación de la agenda de gobierno del presidente de la República. Particularmente, el presidente León Febres Cordero presentó un total de 15 leyes catalogadas como económicas urgentes entre el 20 de septiembre de 1984 y el 5 de agosto de 1985. Sin embargo, es necesario considerar que, durante este periodo, a pesar de la utilización de mecanismos institucionales tendientes a mejorar la posibilidad de aprobación de los proyectos de ley remitidos desde el ejecutivo, el presidente Febres Cordero no consiguió un porcentaje de aprobación grande dentro de la Asamblea Legislativa durante el primer año de su gobierno. No obstante, esta lógica no pudo aplicarse de forma permanente durante su gobierno, debido a que, en instancias posteriores del mismo, un congreso más articulado pudo articular mecanismos de defensa en contra de este procedimiento, impidiendo la aprobación de toda la normativa remitida a la legislatura desde el ejecutivo.

Otro caso interesante ocurre durante el gobierno del presidente Gustavo Noboa, debido a que llegó a la Presidencia como consecuencia del derrocamiento de Jamil Mahuad Witt. Durante el ejercicio de su cargo, el mandatario no se encontró afiliado a ningún partido político, por lo que no contó con una mayoría propia dentro de la legislatura. Por este motivo, como puede evidenciarse en el cuadro que precede, optó por la conformación de mayorías móviles para la aprobación de proyectos de ley considerados fundamentales para su gobierno. De esta forma, a pesar de no contar con una 
mayoría legislativa propia, el presidente Noboa consiguió aprobar de forma exitosa las denominadas leyes Trole I y II $^{5}$. Estas normas, cuyos nombres reales fueron Ley de Transformación Económica del Estado y Ley para la Promoción de la Inversión y de la Participación Ciudadana, respectivamente, generaron sendas reformas a la estructura económica del Estado. Por una parte, la Ley Trole I reguló la implementación de la dolarización en el Ecuador. Por otra parte, la denominada Ley Trole II introdujo modificaciones a 31 cuerpos normativos distintos. En el caso de ambas leyes, ambas fueron catalogadas como económicas urgentes, lo cual, conjuntamente con la obtención de una mayoría móvil dentro de la Asamblea, habría permitido su aprobación.

La estrategia relativa a la utilización de mecanismos institucionales - designación del proyecto de ley como económico urgente - también fue utilizada por Jamil Mahuad Witt desde el mes de marzo de 1999 hasta la finalización de su periodo de funciones. No obstante, en su caso, el mecanismo tuvo algo más de éxito que en el caso de aplicación de la estrategia por parte del presidente Febres Cordero. En todo caso, la situación económica durante el periodo presidencial de Mahuad fue harto compleja, debido a que durante el año 1999, el PIB ecuatoriano cayó en un 4,74\%, siendo la caída económica más grande el país desde el año 1961. En este sentido, la estrategia de Mahuad pudo no tener como objetivo la utilización de un mecanismo institucional encaminado a legislar de manera directa, sino que, pudo responder a la necesidad de tomar medidas drásticas destinadas a dar estabilidad la economía del país.

Por otra parte, dentro del periodo analizado, la producción legislativa de los presidentes de la república ha sido diversa, evidenciando que sus porcentajes de éxito legislativo, en los términos previstos por Mercedes García Montero (2009), han sido variados. No obstante, se debe tomar en cuenta que el porcentaje de éxito legislativo no observa la naturaleza de cada uno de los proyectos de ley remitidos por cada ejecutivo, sin embargo, es una buena medida para tener una imagen general de la capacidad efectiva de cada uno de los presidentes de controlar agenda legislativa. Desde esta perspectiva, a continuación, se exponen los porcentajes de éxito legislativo de cada uno de los presidentes del Ecuador desde el año 1979:

\footnotetext{
5 Este tipo de leyes son denominadas "leyes ómnibus" en otros países. Se les da esta denominación debido a que abarcan una gran cantidad de tópicos, vulnerando, inclusive, las prohibiciones contempladas a nivel constitucional y legal, que establecen la prohibición de que un proyecto de ley se refiera a más de una materia.
} 
Tabla 2: Porcentaje de éxito legislativo de los presidentes ecuatorianos desde 1979

\begin{tabular}{|l|c|c|c|}
\hline \multicolumn{1}{|c|}{ Presidente } & $\begin{array}{c}\text { Proyectos de } \\
\text { Ley presentados }\end{array}$ & $\begin{array}{c}\text { Proyectos de } \\
\text { Ley aprobados }\end{array}$ & $\begin{array}{c}\text { Porcentaje de } \\
\text { éxito legislativo }\end{array}$ \\
\hline Jaime Roldós Aguilera & 38 & 6 & $15,79 \%$ \\
\hline Oswaldo Hurtado Larrea & 78 & 8 & $10,26 \%$ \\
\hline León Febres Cordero & 77 & 23 & $29,87 \%$ \\
\hline Rodrigo Borja Cevallos & 70 & 37 & $52,86 \%$ \\
\hline Sixto Durán Ballén & 84 & 40 & $47,62 \%$ \\
\hline Abdalá Bucaram Ortiz & 10 & 2 & $20 \%$ \\
\hline Fabián Alarcón Rivera & 48 & 33 & $68,75 \%$ \\
\hline Jamil Mahuad Witt & 24 & 10 & $41,67 \%$ \\
\hline Gustavo Noboa Bejarano & 43 & 25 & $58,14 \%$ \\
\hline Lucio Gutiérrez Borbúa & 28 & 7 & $56 \%$ \\
\hline $\begin{array}{l}\text { Alfredo Palacio } \\
\text { González }\end{array}$ & 25 & 14 & $57,14 \%$ \\
\hline $\begin{array}{l}\text { Rafael Correa Delgado } \\
\text { (Primer periodo) }\end{array}$ & 28 & 16 & $66,67 \%$ \\
\hline $\begin{array}{l}\text { Rafael Correa Delgado } \\
\text { (Segundo periodo) }\end{array}$ & 54 & 36 & $66,67 \%$ \\
\hline $\begin{array}{l}\text { Rafael Correa Delgado } \\
\text { (Tercer periodo) }\end{array}$ & 39 & 26 & \\
\hline
\end{tabular}

Fuente: elaboración propia con base en la información disponible en la web: www.asambleanacional.gob.ec

Como se puede verificar en los datos que se exponen de forma previa, las lógicas de aprobación de leyes remitidas desde la función ejecutiva han sido harto diversas. Por una parte, se identifica presidentes, como por ejemplo, Jaime Roldós, Oswaldo Hurtado y Abdalá Bucaram Ortiz que han obtenido porcentajes de éxito legislativo bastante discretos a lo largo de su gestión. Por otra parte, existen casos, como el del presidente Fabián Alarcón, que, además de ser un presidente encargado, y no contar con una bancada legislativa mayoritaria, obtuvo a aprobación del $68,75 \%$ de los proyectos de ley que remitió a la legislatura. Esta lógica, precisamente, permite confirmar que existen otras explicaciones para determinar la capacidad efectiva del ejecutivo para aprobar sus proyectos de ley. En este mismo sentido, puede verificarse una lógica similar en el caso del presidente Alfredo Palacios. Este mandatorio, por su parte, sin contar con una bancada legislativa propia, debido a su ascenso al poder tras el derrocamiento del presidente Lucio Gutiérrez Borbúa, consiguió aprobar un total del 56\% de sus proyectos de ley remitidos a la legislatura.

Curiosamente, puede identificarse que un presidente interino, sin una bancada legislativa propia, fue el presidente con un mayor nivel de éxito legislativo desde el año 1979. Esto confirma que, si bien una bancada 
mayoritaria en la legislatura es beneficiosa para la aprobación de los proyectos de ley remitidos desde el ejecutivo, no es una condición indispensable. En estos casos, evidentemente, se colige la existencia de elementos distintos de carácter explicativo, que fungen como motivo para que se aprueben los proyectos de ley.

Lo descrito nos permite confirmar a través de la observación de los datos recopilados, que si bien, la mayoría legislativa es un elemento importante para explicar por qué unos presidentes consiguen la aprobación de sus proyectos de ley dentro de la legislatura, como en el caso del presidente Correa durante sus dos últimos periodos de mandato, en otros, como los descritos en el párrafo anterior, este requisito no es indispensable para que los presidentes consigan aprobar una cantidad importante de las leyes que remiten a la legislatura.

Este caso se formula únicamente a manera de ejemplo respecto del nivel de volatilidad existente dentro de la legislatura ecuatoriana. Y es por este motivo que, a partir del año 1979, existe una variación interesante de los elementos que se proponen como explicativos para que el presidente consiga la aprobación de los proyectos de ley que remite a la legislatura. Esta cantidad de elementos permiten verificar de forma precisa cuáles son los elementos que deberán estar presentes para que el titular del ejecutivo consiga aprobar sus proyectos de ley dentro de la asamblea.

Pero además se evidencia que la mayoría legislativa del ejecutivo ha cambiado de forma importante durante el periodo a ser analizado, también se han modificado los partidos que lo conforman. Por ejemplo, durante el año 2007 surge el movimiento Alianza País, que ha dominado la escena política nacional desde su creación en términos de número de legisladores ${ }^{6}$. Mientras que, otros partidos, anteriormente importantes, han disminuido su protagonismo de forma superlativa, como los casos del Partido Social Cristiano, la Democracia Popular y la Izquierda Democrática.

Este cambio en las mayorías legislativas, precisamente, nos permite observar la importancia de analizar la forma en que se ha desarrollado la actividad legislativa de los presidentes, debido a que, como puede verificarse, por lo general, ningún presidente, exceptuando el caso de Rafael Correa Delgado, ha contado con una mayoría legislativa propia. Aun así, se debe tomar en cuenta que han sido varios los titulares del ejecutivo que han conseguido formar mayorías legislativas de coalición.

\footnotetext{
${ }^{6}$ Debe exceptuarse de esta definición el primer periodo del presidente Rafael Correa, dado que en un inicio no contó con una bancada legislativa propia. Esta cuestión cambia una vez que se convoca a la Asamblea Nacional Constituyente, a partir del año 2007.
} 
Por otra parte, con relación al nivel de fragmentación partidista, es necesario identificar el número de partidos presentes dentro de la legislatura ecuatoriana. Bajo esta lógica, se puede verificar que la legislatura ha experimentado importantes índices de fragmentación a lo largo de su historia, contando con la presencia de una gran cantidad de partidos políticos con representación en la legislatura. No obstante, a pesar de que han existido gran cantidad de bancadas legislativas, en la mayoría de casos, se verifica la existencia de muchos partidos con poca representación en la legislatura. Siguiendo la propuesta teórica del presente trabajo, ante un legislativo muy fragmentado, la capacidad efectiva del presidente para aprobar sus proyectos de ley debería disminuir. Verificamos esto comparando la filiación política del presidente con el número de partidos dentro de la legislatura:

Tabla 3: Número de partidos en la legislatura 1979-2016

\begin{tabular}{|c|c|c|c|}
\hline Presidente & Período Legislativo & $\begin{array}{l}\text { Partido del } \\
\text { presidente }\end{array}$ & $\begin{array}{c}\mathrm{N}^{\circ} \text { de } \\
\text { partidos en } \\
\text { el Congreso }\end{array}$ \\
\hline Jaime Roldós Aguilera & \multirow{2}{*}{$10-8-1979$ a $9-8-1984$} & CFP & \multirow{2}{*}{11} \\
\hline Oswaldo Hurtado Larrea & & UDC-DP & \\
\hline \multirow{2}{*}{ León Febres Cordero } & 10-8-1984 a 9-8-1986 & \multirow{2}{*}{ PSC } & 13 \\
\hline & 10-8-1986 a 9-8-1988 & & 13 \\
\hline \multirow{2}{*}{ Rodrigo Borja Cevallos } & 10-8-1988 a 9-8-1990 & \multirow{2}{*}{ ID } & 11 \\
\hline & 10-8-1990 a 9-8-1992 & & 12 \\
\hline \multirow{2}{*}{ Sixto Durán Ballén } & 10-8-1992 a 9-8-1994 & \multirow{2}{*}{ PUR-PC } & 12 \\
\hline & 10-8-1994 a 9-8-1996 & & 12 \\
\hline Abdalá Bucaram Ortiz & \multirow{2}{*}{$10-8-1996$ a $9-8-1998$} & PRE & \multirow{2}{*}{11} \\
\hline Fabián Alarcón Rivera & & FRA & \\
\hline Jamil Mahuad Witt & \multirow{2}{*}{ 10-8-1998 a 4-1-2003 } & UDC-DP & \multirow{2}{*}{12} \\
\hline Gustavo Noboa Bejarano & & - & \\
\hline Lucio Gutiérrez Borbúa & \multirow{2}{*}{$5-1-2003$ a 4-1-2007 } & PSC & \multirow{2}{*}{17} \\
\hline Alfredo Palacio González & & - & \\
\hline \multirow{3}{*}{ Rafael Correa Delgado } & 5-1-2007 a 29-11-2007 & \multirow{3}{*}{ MP } & 13 \\
\hline & 30-11-2007 a 27-10-2008* & & 13 \\
\hline & $28-10-2008$ a $30-7-2009^{* *}$ & & 12 \\
\hline Rafael Correa Delgado & $31-7-2009$ a $13-5-2013$ & MP & 19 \\
\hline Rafael Correa Delgado & $14-5-2013$ a 13-5-2017 & MP & 11 \\
\hline
\end{tabular}

* Asamblea Constituyente. ** Comisión Legislativa

Fuente: Rowland \& Burbano de Lara (1998) y Valdivieso (2015) 
Como puede evidenciarse, la legislatura ecuatoriana ha contado con un número importante de partidos a lo largo de su existencia. Este fenómeno, en primera instancia parece explicar el incremento en la capacidad efectiva de aprobación de las leyes remitidas desde el ejecutivo a la legislatura. Por ejemplo, Fabián Alarcón, sin contar con una bancada legislativa grande, consiguió aprobar una importante cantidad de los proyectos de ley que remitió a la legislatura. Esto, por parte, puede ser explicado puesto que en el congreso existían 11 partidos con representación política, y no existió uno solo que cuente con una mayoría suficiente para marcar por su cuenta la agenda legislativa (PSC: 30,49\%; PRE: 24,39\%; UDC: $15,85 \%$ y partidos minoritarios). En este sentido, a pesar de que el nivel de fragmentación fue aparentemente alto, las negociaciones del presidente Alarcón pudieron enfocarse en negociar con los 3 partidos mayoritarios de la Asamblea, consiguiendo de esta forma una cantidad de votos suficientes. Por tanto, la fragmentación, debido a la minoritaria participación de los demás partidos, el número efectivo de partidos, según el indicador generado por Laakso y Taagepera (1979) tenderá a ser más bajo, debido a que el peso efectivo de los tres partidos principales es capturado por este indicador.

Por otra parte, con relación al gobierno del presidente Palacio, se puede verificar que el nivel de fragmentación en este caso fue inclusive más alto, contando con un número total de 17 partidos dentro de la legislatura. Esta situación, de hecho, podría aducirse que el mayor nivel de dispersión existente dificultaría la posibilidad de aprobar los proyectos de ley del ejecutivo. No obstante, durante este periodo, a pesar de que existió un gran número de partidos en el congreso, solo tres partidos contaron con una bancada legislativa representativa: PSC: $25 \%$; ID: $16 \%$ y PRE: $15 \%$. Pero además, dos partidos contaron con una bancada que, aunque no es tan representativa como las antes enunciadas, sí se constituye como una mayoría lo suficientemente grande como tener algún nivel de incidencia dentro de la toma de decisiones en la legislatura. Estos partidos fueron el PRIAN y el PSP, con representación legislativa del $10 \%$ y $9 \%$, respectivamente. En cualquier caso, a pesar de la existencia de una gran cantidad de partidos, el hecho de que solo algunos cuenten con un porcentaje de asientos importante también puede facilitar la existencia de procesos de negociación, que, a su vez, permitan al ejecutivo la aprobación de su agenda legislativa.

Esto podría llevarnos a pensar que la fragmentación partidista como tal no se constituye necesariamente como una variable de peso. No obstante, para detectar una clara correlación o ausencia de ella, debemos implementar futuros estudios que aporten con métodos estadísticos más desarrollados.

En este sentido, debe considerarse que, según el trabajo de Mejía (2004), en muchos casos pueden existir coaliciones fantasmas, formadas a 
través de pagos ilegítimos y transferencia de recursos a los legisladores a cambio de votos. En estos casos, la explicación para la aprobación de un proyecto de ley podría encontrarse en este tipo de mecanismos sin que le valor de las variables codificadas puede capturar este tipo de información. Este supuesto, cabe aclarar, podría suscitarse en legislaturas de carácter parroquial venal, en los términos previstos por Cox y Morgenstern (2001). Sin embargo, a pesar de que esta observación no puede efectuarse de manera directa, es importante determinar una agenda de investigaciones para su realización futura, precisamente, considerado este tipo de elementos.

Por su parte, en cuanto al posicionamiento ideológico, variable de orden político, de cada uno de los ejecutivos, se evidencia la existencia de valores bastante distintos, sin que haya existido otro ejecutivo catalogado como de izquierda distinta a Rafael Correa Delgado. Se le acerca Rodrigo Borja Cevallos, quien cuenta con un valor más cercano a uno. No obstante, su posición es más tendiente al centro. Por cuestiones relativas a la naturaleza de los datos disponibles, ha sido necesario imputar valores de periodos distintos a periodos anteriores en los cuales dicha información no ha sido medida. Sin embargo, los datos correspondientes de posicionamiento ideológico de cada uno de los ejecutivos en el periodo de tiempo analizado, con la salvedad enunciada, son los siguientes:

Tabla 4: Posicionamiento ideológico de los ejecutivos

\begin{tabular}{|l|c|}
\hline \multicolumn{1}{|c|}{ Presidente } & $\mathbf{N}^{\circ}$ de partidos en el Congreso \\
\hline Jaime Roldós Aguilera & 4,5 \\
\hline Oswaldo Hurtado Larrea & 5,23 \\
\hline León Febres Cordero & 8,72 \\
\hline Rodrigo Borja Cevallos & 4,87 \\
\hline Sixto Durán Ballén & 7,5 \\
\hline Abdalá Bucaram Ortiz & 6,34 \\
\hline Fabián Alarcón Rivera & Sin información \\
\hline Jamil Mahuad Witt & 6,94 \\
\cline { 1 - 1 } Gustavo Noboa Bejarano & 7 \\
\hline Lucio Gutiérrez Borbúa & 5,82 \\
\hline Alfredo Palacio González & Sin información \\
\cline { 1 - 2 } Rafael Correa Delgado & 2,9 (primer periodo) \\
\cline { 1 - 2 } & 3,4 (segundo periodo) \\
\hline
\end{tabular}

Elaboración propia con base en la información del proyecto de élites parlamentarias de la Universidad de Salamanca ${ }^{7}$

7 El dato de posicionamiento ideológico ha sido obtenido del proyecto de élites parlamentarias, llevado a cabo por la Universidad de Salamanca. Este proyecto determina 
Sin detrimento de los esfuerzos efectuados con el fin de obtener datos completos relativos a la posición ideológica de cada uno de los ejecutivos, no ha sido posible conseguir un dato fiable para Fabián Alarcón y Alfredo Palacio. Esto debido a que accedieron al poder de forma posterior a la renuncia de los titulares del ejecutivo. Por lo tanto, durante estos periodos, inclusive, ambos presidentes no contaron con una bancada legislativa importante, y sus gobiernos podrían haberse basado en posiciones netamente pragmáticas que les permitan una adecuada negociación, con el fin de permanecer en el poder.

Se verifica una fuerte modificación de los valores correspondientes a la ideología de cada uno de los ejecutivos dentro de la Asamblea Legislativa. En este caso, precisamente, esta heterogeneidad demuestra de forma efectiva la gran cantidad de cambios existentes, lo cual permite de forma efectiva analizar los efectos de la varianza de esta variable explicativa contestando la pregunta de investigación propuesta.

Por su parte, el análisis de la naturaleza de las leyes propuestas por cada uno de los ejecutivos es un factor importante para determinar, además de las lógicas relativas a su éxito legislativo, la naturaleza de los proyectos de ley que cada uno de ellos consiguió aprobar.

Desde este punto de vista, podemos verificar que, por una parte, las leyes orgánicas no fueron materia de propuesta del ejecutivo en términos mayoritarios sino hasta el gobierno de Rafael Correa. Por otra parte, en cuanto a las materias y la importancia, se verifica un importante nivel de cambio entre cada ejecutivo. ${ }^{8}$

Por otra parte, para el presente ejercicio se ha categorizado a los proyectos de ley de acuerdo a dos criterios. El primero, la materia a la cual este se refiere, generando las categorías: económica, política, social y otras. El segundo, su relevancia, de acuerdo a los previsto por Mayhew (1991). En concreto, este autor propone tres categorías para clasificar leyes, que son las siguientes: leyes hito (aquellas que crean una institución), leyes importantes (aquellas que reforman una ley hito), leyes no importantes (las que no ocupan ninguna de las dos categorías anteriores).

una serie de datos vinculados a la legislatura de los países latinoamericanos. Entre los múltiples datos que aporta, se encuentra el posicionamiento ideológico, mismo que es calculado en una escala del 1 (izquierda) al 10 (derecha), en base a encuestas realizadas a expertos de cada uno de los países analizados. La información puede consultarse y verificarse en: http://americo.usal.es/oir/elites/

${ }^{8}$ Para una correcta lectura de este punto, es necesario considerar que las constituciones ecuatorianas no contemplaron una distinción entre leyes ordinarias y orgánicas sino hasta la Constitución de 1998, cuyo artículo 142 recogió esta distinción. La actual Constitución, promulgada durante el año 2008, replica esta distinción dentro de su artículo 133. 
Los datos completos son los siguientes:

Tabla 5: Tipos de leyes aprobadas

\begin{tabular}{|c|c|c|c|c|c|c|c|c|c|c|c|}
\hline 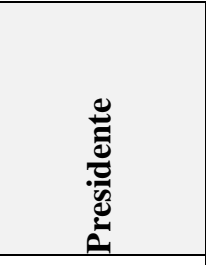 & 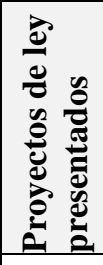 & 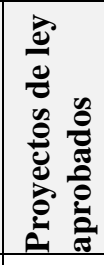 & 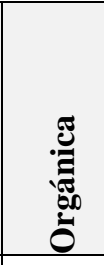 & 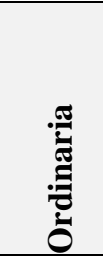 & 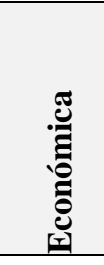 & 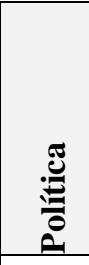 & 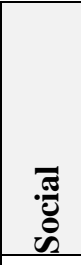 & $\stackrel{\pi}{0}$ & 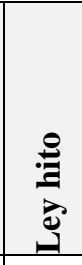 & 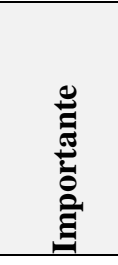 & 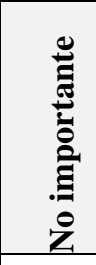 \\
\hline $\begin{array}{l}\text { Jaime Roldós } \\
\text { Aguilera }\end{array}$ & 38 & 6 & $1(0)$ & 37 (6) & $21(4)$ & $2(0)$ & $\begin{array}{l}12 \\
(2) \\
\end{array}$ & \begin{tabular}{|l|}
3 \\
$(0)$ \\
\end{tabular} & $\begin{array}{l}10 \\
(2) \\
\end{array}$ & $23(4)$ & $5(0)$ \\
\hline $\begin{array}{l}\text { Oswaldo } \\
\text { Hurtado L. }\end{array}$ & 78 & 8 & $4(1)$ & 74 (7) & 27 (2) & \begin{tabular}{|l|}
11 \\
$(1)$
\end{tabular} & \begin{tabular}{|l|}
29 \\
$(4)$
\end{tabular} & $\begin{array}{l}11 \\
(1)\end{array}$ & $\begin{array}{l}16 \\
(1)\end{array}$ & $48(6)$ & $14(1)$ \\
\hline $\begin{array}{l}\text { León Febres } \\
\text { Cordero }\end{array}$ & 77 & 23 & $4(2)$ & $\begin{array}{l}73 \\
(21) \\
\end{array}$ & $\begin{array}{l}31 \\
(13) \\
\end{array}$ & $5(0)$ & $\begin{array}{l}27 \\
(5) \\
\end{array}$ & \begin{tabular}{|l|l}
14 \\
$(5)$ \\
\end{tabular} & \begin{tabular}{|l|}
7 \\
$(1)$ \\
\end{tabular} & $46(14)$ & $24(8)$ \\
\hline $\begin{array}{l}\text { Rodrigo } \\
\text { Borja C. }\end{array}$ & 70 & 37 & $5(2)$ & $\begin{array}{l}65 \\
(35) \\
\end{array}$ & $\begin{array}{l}20 \\
(10)\end{array}$ & 7 (3) & \begin{tabular}{|l|}
20 \\
$(9)$
\end{tabular} & \begin{tabular}{|l|}
23 \\
$(15)$ \\
\end{tabular} & $\begin{array}{l}18 \\
(9)\end{array}$ & 26 (14) & \begin{tabular}{|l|}
26 \\
$(14)$
\end{tabular} \\
\hline $\begin{array}{l}\text { Sixto Durán } \\
\text { Ballén }\end{array}$ & 84 & 40 & $5(2)$ & $\begin{array}{l}79 \\
(38) \\
\end{array}$ & $\begin{array}{l}34 \\
(17)\end{array}$ & $6(2)$ & $\begin{array}{l}126 \\
(12) \\
\end{array}$ & $\begin{array}{l}18 \\
(9) \\
\end{array}$ & $\begin{array}{l}21 \\
(8)\end{array}$ & $45(22)$ & \begin{tabular}{|l|}
18 \\
$(10)$
\end{tabular} \\
\hline $\begin{array}{l}\text { Abdalá } \\
\text { Bucaram O. }\end{array}$ & 10 & 2 & 0 & $10(2)$ & $8(2)$ & 0 & \begin{tabular}{|l|}
1 \\
$(0)$
\end{tabular} & \begin{tabular}{|l}
1 \\
$(0)$
\end{tabular} & \begin{tabular}{|l|}
3 \\
$(1)$ \\
\end{tabular} & $5(1)$ & $2(0)$ \\
\hline $\begin{array}{l}\text { Fabián } \\
\text { Alarcón R. }\end{array}$ & 48 & 33 & $4(1)$ & $\begin{array}{l}44 \\
(32)\end{array}$ & $\begin{array}{l}27 \\
(19)\end{array}$ & $3(1)$ & \begin{tabular}{|l|}
11 \\
$(10)$
\end{tabular} & \begin{tabular}{|l|}
7 \\
$(2)$
\end{tabular} & \begin{tabular}{|l|}
15 \\
$(11)$
\end{tabular} & $26(18)$ & $7(4)$ \\
\hline $\begin{array}{l}\text { Jamil } \\
\text { Mahuad Witt }\end{array}$ & 24 & 10 & 0 & $\begin{array}{l}24 \\
(10)\end{array}$ & $16(7)$ & $3(0)$ & \begin{tabular}{|l|}
4 \\
$(3)$
\end{tabular} & $\begin{array}{l}1 \\
(0)\end{array}$ & \begin{tabular}{|l|}
5 \\
$(1)$
\end{tabular} & $16(7)$ & $3(2)$ \\
\hline $\begin{array}{l}\text { Gustavo } \\
\text { Noboa B. }\end{array}$ & 43 & 25 & $8(4)$ & $\begin{array}{l}35 \\
(21)\end{array}$ & $\begin{array}{l}21 \\
(12)\end{array}$ & $4(2)$ & $\begin{array}{l}9 \\
(5)\end{array}$ & $\begin{array}{l}9 \\
(6) \\
\end{array}$ & \begin{tabular}{|l}
9 \\
$(5)$ \\
\end{tabular} & $23(13)$ & $11(7)$ \\
\hline $\begin{array}{l}\text { Lucio } \\
\text { Gutiérrez B. }\end{array}$ & 28 & 7 & $8(4)$ & $20(3)$ & $9(2)$ & $1(0)$ & $\begin{array}{l}9 \\
(4)\end{array}$ & $\begin{array}{l}9 \\
(1)\end{array}$ & \begin{tabular}{|l}
5 \\
$(1)$ \\
\end{tabular} & $14(5)$ & $9(1)$ \\
\hline $\begin{array}{l}\text { Alfredo } \\
\text { Palacio G. }\end{array}$ & 25 & 14 & $13(6)$ & $12(8)$ & $9(8)$ & $8(2)$ & \begin{tabular}{|l}
6 \\
$(3)$
\end{tabular} & $\begin{array}{l}2 \\
(1)\end{array}$ & \begin{tabular}{|l}
8 \\
$(3)$ \\
\end{tabular} & $14(10)$ & $3(1)$ \\
\hline $\begin{array}{l}\text { Rafael } \\
\text { Correa D. } \\
\text { (periodo 1) }\end{array}$ & 121 & 78 & \begin{tabular}{|l}
51 \\
$(29)$
\end{tabular} & $\begin{array}{l}70 \\
(49)\end{array}$ & $\begin{array}{l}42 \\
(30)\end{array}$ & $\begin{array}{l}27 \\
(17)\end{array}$ & \begin{tabular}{|l}
36 \\
$(22)$
\end{tabular} & \begin{tabular}{|l}
16 \\
$(9)$
\end{tabular} & \begin{tabular}{|l}
49 \\
$(33)$
\end{tabular} & 60 (39) & $12(6)$ \\
\hline
\end{tabular}

Fuente: elaboración propia

Nota: el número en paréntesis corresponde al número de proyectos aprobados.

En sentido coyuntural, es conveniente analizar que la aprobación o no de los proyectos de ley remitidos por parte del ejecutivo a la legislatura puede depender de elementos de carácter coyuntural. Dentro del presente estudio, se toma el crecimiento del PIB como un indicador proxy para determinar la favorabilidad del contexto para aprobación de las iniciativas legislativas remitidas desde el ejecutivo. En este sentido, se entenderá que, durante momentos de auge económico, será más probable que el presidente 
apruebe sus proyectos de ley en la legislatura. Esto, según el supuesto teórico manejado, sucedería por dos motivos: la situación económica favorable permitiría que el presidente mejore su nivel de aceptación, por tanto, contará con un mayor respaldo popular que le permitirá ejercer presión sobre la legislatura; y, en una situación económica favorable, existen más recursos que pueden ser destinados a efectuar pagos ilegítimos o concesiones presupuestarias a favor de legisladores que podrían destinar su voto a la aprobación de las iniciativas legislativas del ejecutivo.

En este sentido, la evolución en el crecimiento del PIB ecuatoriano desde el año 1978 ha sido la siguiente:

Figura 1: Evolución del PIB ecuatoriano 1979 - 2016

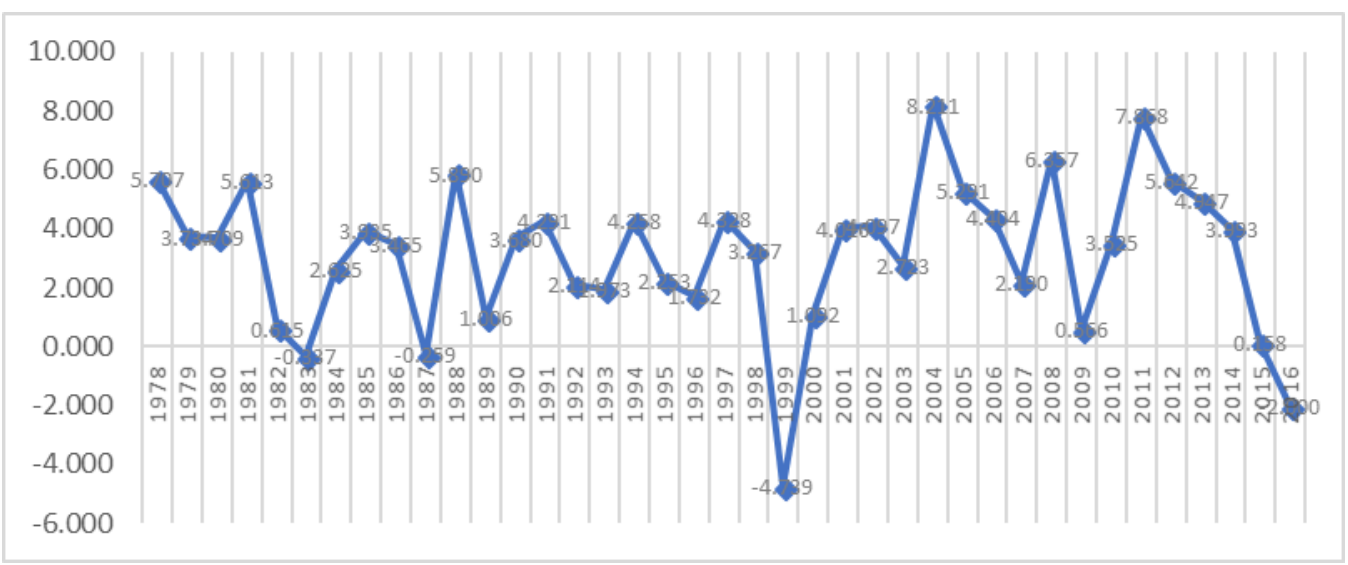

Fuente: elaboración propia con base en los datos obtenidos en datos.bancomundial.org

Por lo tanto, podría preverse que, durante los años en los que se suscita un crecimiento del PIB, los presidentes afrontan una coyuntura más favorable para la aprobación de sus proyectos de ley. Bajo esta perspectiva, podría presumirse que, durante los años 1983, 1987, 1999 y 2016 los presidentes ecuatorianos experimentaron un contexto más complejo para la aprobación de sus iniciativas legales remitidas a la legislatura. Por otra parte, durante los años 1988, 2004 y 2011, puede pensarse que la coyuntura fue más favorable para la aprobación de los proyectos de ley remitidos desde la función ejecutiva a la legislatura por las condiciones favorables de la economía. Por lo tanto, aunque no se cuenta con datos suficientes para determinar una inferencia causal clara, es evidente que el contexto favorable en el ámbito social y económico incrementa la capacidad del ejecutivo de aprobar os proyectos de ley remitidos a la legislatura.

En todo caso, los datos obtenidos a través de este mecanismo no pueden dar luces claras sobre la influencia de variables contextuales de 
forma íntegra, pues existen muchas otras que pudieran ser influyentes para determinar la capacidad de un ejecutivo de aprobar sus proyectos de ley.

\section{CONCLUSIONES Y AGENDA}

1. Como puede evidenciarse de los datos consignados anteriormente, existen varios patrones que resultan de análisis interesante. En primer lugar, de la simple observación se deduce que no existe un patrón claro de mayoría parlamentaria para obtener la aprobación de los proyectos de ley. Así, existen casos como el del presidente Alarcón, que sin contar con una bancada legislativa, consiguió aprobar más del $60 \%$ de sus proyectos de ley. Esto da cuenta de que, aunque la mayoría en la legislatura es efectivamente relevante para incrementar la posibilidad de que un presidente apruebe sus proyectos de ley, no es un factor único y exclusivo.

2. Por otra parte, no se verifica que las variables fragmentación partidista y distancia ideológica sean relevantes al momento de explicar el por qué el ejecutivo consigue aprobar sus proyectos de ley. Esto podría explicarse por el hecho de que los partidos presentes en la Asamblea son de carácter parroquial venal, o depredador, en términos de Cox y Morgenstern (2001). Evidentemente el plan de un actor de esta naturaleza no tendrá mayor apego a una postura ideológica ni acatará cuestiones de fragmentación partidista, ya que su objetivo será básicamente conseguir réditos propios o para su propia clientela. Sin embargo, en legislaturas mayormente institucionalizadas, una fragmentación partidista alta (muchos partidos en la legislatura), implica que las aristas de negociación serán mayores y más complejas, incrementando los costos de iniciar negociaciones por parte del ejecutivo. Por su parte, la distancia ideológica, en general, a nivel teórico, es relevante, ya que mientras más lejanos ideológicamente sean dos actores políticos, será más complejo que puedan acordar en un punto medio, que resulte satisfactorio para ambos. Por lo tanto, se puede inferir que a menor distancia ideológica entre el ejecutivo y quienes conforman la legislatura, será más probable que un presidente apruebe sus proyectos de ley.

3. Por otra parte, se verifica que, aunque las variables de contexto son aparentemente beneficioso para que un ejecutivo apruebe su agenda ante la legislatura, esto no ocurre siempre. Por lo tanto, es posible que el valor del crecimiento del PIB no se constituya como una variable de contexto que otorgue una visión general de la situación. No obstante, a través de la observación de los datos recopilados, se puede observar que un contexto económico favorable, así como circunstancias políticas favorables, podrían beneficiar al ejecutivo en la aprobación de sus proyectos de ley. 
4. Por otra parte, no se constata que la materia del proyecto de ley sea altamente relevante. De hecho, se puede observar claramente que los presidentes han logrado aprobar todo tipo de leyes sin que exista un patrón claro que permita dar prioridad a una determinada materia de proyectos de ley. Igualmente, en el ámbito de la importancia de un proyecto de ley, a partir de las características enunciadas en fases previas del presente estudio, se verifica un mayor nivel de relevancia. Esto da cuenta de que las categorías presentadas por Mayhew (1991) son valederas y se verifican con diferencias en el rendimiento empírico. Por otra parte, el criterio institucional no es un parámetro adecuado para medir, debido a la mala concepción de la reserva de ley orgánica y ordinaria existente en el Ecuador. E inclusive, considerando que en el Ecuador existe la distinción entre ley orgánica y ley ordinaria a partir del año 1998.

5. Por otra parte, se verifica que las capacidades institucionales del ejecutivo en efecto fungen como un poderoso elemento de negociación a partir del cual el presidente puede configurar su forma de proceder de acuerdo con los cuatro supuestos que fueron considerados de forma inicial dentro de este estudio. De esta forma, podríamos asumir que generar una predicción estática sobre lo que convierte a un presidente en exitoso en el ámbito legislativo no necesariamente puede responder a una fórmula exacta, sino que, su estrategia dependerá de un cálculo inicial de las atribuciones con las que cuenta.

6. Esta información nos permite observar de forma específica que la determinación de elementos comunes que expliquen el éxito de los ejecutivos es compleja en el ámbito del Ecuador. Este estudio genera una apuesta a futuro importante, dado que implica la necesidad de verificar las hipótesis propuestas por la literatura, constatando inclusive la necesidad de incurrir en nuevos modelos metodológicos que permitan analizar las cuestiones minuciosas que no son observables a través de estudios con un número grande de unidades de análisis. 


\section{REFERENCIAS}

Alemán, E. \& Navia, P. (2009). Institutions and the Legislative Success of "Strong" Presidents: An Analysis of Government Bills in Chile. The Journal of Legislative Studies, 1, pp. 401-419.

Amorim Neto, O. \& Cox, G. (1998). Electoral institutions, cleavage structures, and the number of parties. American Journal of Political Science, 41(1), pp. 149-174.

Amorim Neto, O., Cox, G. \& McCubbins, M. (2003). Agenda power in Brazil's Câmara dos Deputados, 1989-98. World Politics, 55(4), pp. 550-578.

Bond, J. \& Fleisher, R. (1990). The President in the Legislative Arena. Chicago, IL: University of Chicago Press.

Burbano de Lara, F. \& Rowland, M. (1998). Pugna de poderes en Ecuador (1979-1997). Quito: Flacso.

Covington, C., Wrighton, M. \& Kinney, R. (1995). A PresidencyAugmented Model of Presidential Success in Roll Call Votes. American Journal of Political Science, 39(4), pp. 1001-1024.

Cox, G. \& Morgenstern, S. (2001). Latin America's Reactive Assemblies and Proactive Presidents. Comparative Politics, 2, pp. 171-89.

Edwards, G. (1989). At the Margins: Presidential Leadership of Congress. New Haven,

García Montero, M. (2007). La actividad legislativa en América Latina: sobre el papel reactivo y proactivo de Presidentes y Parlamentos. Lateinamerika Analysen, 17(2), pp. 3-24.

García Montero, M. (2009). Presidentes y Parlamentos ¿quién controla la actividad legislativa en América Latina? Madrid: Centro de Investigaciones Sociológicas.

Linz, J. (1990). The Perils of Presidentialism. Journal of Democracy, 1(1), pp. 51-69.

Mainwaring, S. \& Shugart, M. (edits.) (1997). Presidentialism and Democracy in Latin America. Cambridge: Cambridge University Press. 
Mayhew, D. (1991). Divided Party Control: Does It Make a Difference? PS: Political Science and Politics, 24(4), pp. 637-640.

Mayhew, D. (2005). Divided We Govern: Party Control, Lawmaking, and Investigations, 1946-2002. New Haven, CT: Yale University Press.

Mejía Acosta, A. (2002). Gobernabilidad Democrática: Sistema Electoral, partidos políticos y pugna de poderes en Ecuador: 1978-1998. Quito: Konrad Adenauer Stiftung.

Mejía Acosta, A. (2004). Ghost Coalitions: Economic Reforms, Fragmented Legislatures and Informal Institutions in Ecuador (1979-2002). Notredame: Notredame University.

Morgenstern, S. \& Nacif, B. (eds.) (2002). Legislative Politics in Latin America. Nueva York: Cambridge University Press.

Negretto, G. (2003a). ¿Gobierna sólo el presidente? Poderes de Decreto y diseño institucional en Brasil y Argentina. Desarrollo Económico, 42(167), pp. 377-404.

Negretto, G. (2003b). Diseño constitucional y separación de poderes en América Latina. Revista Mexicana de Sociología, 65(1), pp. 41-76.

Nino, C. (1996). Hyper-Presidentialism and Constitutional Reform. En A. Lijphart \& C. Waisman (eds.), Institutional Design in New Democracies. Boulder: Westview.

O’Donnell, G. (1994). Delegative Democracy. Journal of Democracy, 5 (1), pp. 55-69.

Saiegh, S. (2010). ¿Jugadores activos o apéndices del ejecutivo? Una evaluación del papel de los legisladores latinoamericanos en la toma de decisiones. Política y Gobierno, 17(1), pp. 3-24.

Santos, M., Pérez-Liñán, A. \& García Montero, M. (2014). El control presidencial de la agenda legislativa en América Latina. Revista de Ciencia Política, 34(3), pp. 511-536.

Shugart, M. \& Carey, J. (1992). Presidents and Assemblies: Constitutional design and electoral dynamics. Cambridge: Cambridge University Press.

Tsebelis, G. \& Alemán, E. (2005). Presidential conditional agenda setting in Latin America. World Politics, 57(1), pp. 396-420. 
Valdivieso, P. (2015). El veto presidencial en el Ecuador. Periodo 19792013. En http://repositorio.puce.edu.ec/handle/22000/10130 (recuperado el 6-5-2018).

Valdivieso, P. \& Rivera, L. (2015). Presidencialismo Fuerte en Ecuador (1979/1998/2008). En R. Oyarte (comp.), Derecho constitucional e instituciones políticas, derechos humanos y justicia constitucional. Quito: CEP, pp. 139-158. 